Check for updates

Cite this: Soft Matter, 2017, 13,7548

Received 14th June 2017. Accepted 20th August 2017

DOI: $10.1039 / \mathrm{c} 7 \mathrm{sm} 01180 f$

rsc.li/soft-matter-journal

\section{Collective sedimentation of squirmers under gravity $\dagger$}

\author{
Jan-Timm Kuhr, (D) * Johannes Blaschke, (D) Felix Rühle and Holger Stark*
}

\section{Introduction}

Microswimmers, whether biological or artificially produced, propel themselves forward without the help of any external force. ${ }^{1}$ Often however, external fields act on active particles. ${ }^{2}$ Examples are microswimmers in shear, ${ }^{3-6}$ Poiseuille, ${ }^{4,7-10}$ or swirling flow, ${ }^{11}$ in harmonic traps, ${ }^{12-15}$ and in light fields. ${ }^{16,17}$ The most natural example is gravity, which affects every swimmer that is not neutrally buoyant. In dilute active suspensions, where hydrodynamic interactions between swimmers can be neglected, both experimental ${ }^{18,19}$ and theoretical studies ${ }^{20,21}$ find exponential density profiles similar to that of passive colloids, but with a sedimentation length $\delta$, which depends on activity and well surpasses that of passive colloids. Interestingly, in these dilute suspensions, analytical studies show the emergence of polar order, ${ }^{21}$ and if the microswimmers are bottom heavy, the sedimentation profile can even be inverted. ${ }^{22}$

For higher densities of microswimmers hydrodynamic interactions become important and collective behavior emerges. ${ }^{2,14,23-29}$ This includes motility-induced phase separation, ${ }^{30-34}$ swarming, ${ }^{16,35}$ and bioconvection, ${ }^{36-39}$ to name but a few phenomena. Furthermore, in real settings interactions with bounding surfaces are important, ${ }^{31,34,40-42}$ especially if the swimmers are not perfectly buoyant. ${ }^{43}$

In this article we consider systems with thousands of microswimmers under the influence of gravity. We simulate their full

Institut für Theoretische Physik, Technische Universität Berlin, Hardenbergstr. 36, 10623 Berlin, Germany.E-mail: jan-timm.kuhr@tu-berlin.de, holger.stark@tu-berlin.de $\dagger$ Electronic supplementary information (ESI) available: 2 video files. See DOI: $10.1039 / \mathrm{c} 7 \mathrm{sm} 01180 \mathrm{f}$ hydrodynamic flow fields using the method of multi-particle collision dynamics (MPCD $)^{44,45}$ in order to include hydrodynamic interactions between swimmers as well as between swimmers and bounding walls. As a model microswimmer we use the squirmer, ${ }^{46-49}$ which is versatile enough to model the relevant swimmer types including pushers, pullers, and neutral swimmers.

In the following we concentrate on the case, where passive colloids would just strongly sediment to the bottom. We show how density or sedimentation profiles depend on the ratio of active to sedimentation velocity as well as on the squirmer type. During collective sedimentation squirmers develop densely packed layers in the bottom region of the simulation cell. In contrast, we observe an exponential density profile in the upper region, where squirmers form a more dilute active suspension. The mean vertical orientation of the squirmers depends strongly on their vertical position as well as on their swimmer type. For swimming velocities larger than the sedimentation velocity, we find that hydrodynamic interactions organize squirmers into convection cells. Importantly, both the strength of convection and the extension of the convection cells depend on the squirmer type.

The article is organized as follows. We first introduce the squirmer as our model microswimmer and then shortly address the simulation method of MPCD along with parameter settings and some details of our analysis in Section 2. In Section 3 we present our results of collectively sedimenting squirmers and analyze especially sedimentation and mean vertical orientation (in Section 3.1) and convection (in Section 3.2). Finally, in Section 4 we summarize our findings and conclude. 


\section{Methods and model}

\subsection{The squirmer as a model swimmer}

In this work we use the squirmer ${ }^{46-49}$ as a model for a spherical microswimmer with radius $R$. It propels itself forward using a slip velocity field on its surface,

$$
\mathbf{v}_{\mathrm{s}}\left(\mathbf{r}_{\mathrm{s}}\right)=B_{1}\left(1+\beta \hat{\mathbf{e}} \cdot \hat{\mathbf{r}}_{\mathrm{s}}\right)\left[\left(\hat{\mathbf{e}} \cdot \hat{\mathbf{r}}_{\mathrm{s}}\right) \hat{\mathbf{r}}_{\mathrm{s}}-\hat{\mathbf{e}}\right],
$$

which generates a hydrodynamic flow field in the surrounding fluid. Here, $\mathbf{r}_{\mathrm{s}}$ is a vector, which points from the center of the squirmer to a point on its surface, $\hat{\mathbf{r}}_{\mathrm{s}}=\mathbf{r}_{\mathrm{S}} / R$ is the corresponding unit vector, and the unit vector $\hat{\mathbf{e}}$ indicates the squirmer orientation. In the bulk of a quiescent fluid the squirmer orientation coincides with its swimming direction. Our model in eqn (1) only takes into account the first two terms introduced in ref. 46 and 47 . They are sufficient to determine the swimming speed and swimmer type, by which microorganisms and artificial microswimmers like Janus particles ${ }^{50,51}$ or active droplets ${ }^{52-57}$ are typically characterized. Thus, the squirmer propels along its orientation vector $\hat{\mathbf{e}}$ with swimming speed $v_{0}=2 / 3 B_{1}$ and creates fluid flow, the far field of which is controlled by the parameter $\beta$. The value of $\beta$ therefore indicates the squirmer type. While $\beta=0$ creates a neutral squirmer with the far field of a source dipole $\left(\sim r^{-3}\right), \beta<0$ and $>0$ refer to pushers or pullers, respectively, the flow fields of which decay like a force dipole $\left(\sim r^{-2}\right) .^{58}$

\subsection{Multi-particle collision dynamics}

We investigate the behavior of many squirmers, which interact hydrodynamically with each other and with confining surfaces. We employ multi-particle collision dynamics (MPCD) $)^{44,45,59-61}$ to numerically solve the Navier-Stokes equations including thermal noise. They reduce to the Stokes equations at the low Reynolds numbers studied in this article.

In our MPCD simulations the fluid is modeled by $c a .2 \times$ $10^{7}$ point particles of mass $m_{0}$. Their positions $\mathbf{r}_{i}$ are updated in a streaming step using their velocities $\mathbf{v}_{i}: \mathbf{r}_{i}(t+\Delta t)=\mathbf{r}_{i}(t)+\mathbf{v}_{i} \Delta t$. In the subsequent collision step fluid particles within cubic cells of linear extension $a_{0}$ exchange momentum according to the MPC-AT + a rule. ${ }^{60}$ This conserves linear and angular momentum and also thermalizes velocities to temperature $T$. Further details of our implementation are described in ref. 31 and 34. We use here the parallelized version of ref. 34 , which is suited to simulate large systems with many swimmers.

In the present work, we consider squirmers under gravity. So we have to add an acceleration term $\mathbf{a} \Delta t^{2} / 2$ to the squirmers' position in the streaming step, where the acceleration a is due to the gravitational force $-m g \mathbf{e}_{z}$ along the vertical with $m$ the buoyant mass of a squirmer and $g$ the gravitational acceleration. Since gravity does not induce a noticeable density change of the fluid on the micron length scale, we do not apply a gravitational acceleration to the fluid particles. If a fluid particle encounters a bounding wall or a squirmer, the particle's position and velocity are updated according to the "bounce-back rule", 62 which implements either the no-slip boundary condition or the surface flow field of eqn (1), respectively. During the streaming step momentum is transferred from the fluid particles to the squirmers, the velocities of which are updated by a molecular dynamics step. This includes steric interactions among squirmers and with bounding walls.

MPCD reliably reproduces the analytical results, including the flow field around passive colloids, ${ }^{59}$ the friction coefficient of a particle approaching a plane wall, ${ }^{63}$ the active velocity of squirmers, ${ }^{49}$ as well as the torque acting on them close to walls, where lubrication theory has to be applied. ${ }^{41}$ It also simulates correctly segregation and velocity oscillations in dense colloidal suspensions under Poiseuille flow. ${ }^{64,65}$ MPCD resolves flow fields on time and length scales large compared to the duration of the streaming step $\Delta t$ and the mean free path of the fluid particles, respectively. Therefore, using a squirmer radius of $R=4 a_{0}$, we expect to resolve hydrodynamic flow fields even when squirmers are close to each other.

\subsection{Parameters}

We simulate the behavior of $N=2560$ squirmers of radius $R=4 a_{0}$ under gravity in a cuboidal box of extensions $L_{x}=$ $L_{y}=112 a_{0}$ in the horizontal plane and $L_{z}=224 a_{0}$ along the vertical. Thus, the mean volume fraction of squirmers amounts to $\phi \approx 0.244$. At $z=0$ and $z=L_{z}$ our system is bounded by walls, while periodic boundary conditions apply in the horizontal directions. For the duration of the streaming step we choose $\Delta t=0.02 a_{0} \sqrt{m_{0} / k_{\mathrm{B}} T}$, which sets the shear viscosity to $\eta=16.05 \sqrt{m_{0} k_{\mathrm{B}} T} / a_{0}{ }^{2} \cdot{ }^{66}$

In the following, an important parameter will be the ratio of active to bulk sedimentation velocity,

$$
\alpha:=\frac{v_{0}}{v_{\mathrm{g}}} .
$$

For spherical squirmers with buoyant mass $m$ and gravitational acceleration $g$ one has $v_{\mathrm{g}}=m g /(6 \pi \eta R)$. We will keep $v_{0}$ fixed (it is set by $v_{0}=2 / 3 B_{1}$ with $B_{1}=0.1 \sqrt{k_{\mathrm{B}} T / m_{0}}$ ), and choose three values of $m g$ in order to study the cases $\alpha=0.3,1.0$, and 1.5. In addition, we mention the sedimentation lengths of passive Brownian particles with the same buoyant masses $\mathrm{m}, \delta_{0}=k_{\mathrm{B}} T /(m g)$, where $k_{\mathrm{B}} T$ is thermal energy. For the values of $\alpha$ given above we calculate from this formula the respective values $\delta_{0}=9.3 \times 10^{-4} R, 3.1 \times 10^{-3} R$, and $4.7 \times 10^{-3} R$, so that without activity squirmers would settle into a dense packing at the bottom of the simulation cell.

The Péclet number $\mathrm{Pe}=v_{0} R / D$, where $D=k_{\mathrm{B}} T /(6 \pi \eta R)$ is the translational diffusion coefficient, has the value $\mathrm{Pe}=323$ in all simulations, thus thermal translational motion is negligible. Furthermore, in all our simulations we have a Reynolds number of $\mathrm{Re}=v_{0} R n_{\mathrm{fl}} / \eta=0.17$, where $n_{\mathrm{fl}}=10$ is the average number of fluid particles per collision cell. This implies Stokesian hydrodynamics where inertia can be neglected. Finally, with the thermal rotational diffusion coefficient $D_{\mathrm{r}}=k_{\mathrm{B}} T /\left(8 \pi \eta R^{3}\right)$, we introduce the persistence number $\mathrm{Pe}_{\mathrm{r}}=v_{0} /\left(D_{\mathrm{r}} R\right)$. It measures the distance in units of particle radius $R$, where the squirmer moves persistently in one direction, before rotational diffusion changes its orientation. For all our simulations we have $\mathrm{Pe}_{\mathrm{r}}=430$. Thus, without gravity a single isolated squirmer would swim across the vertical extent $L_{z}$ of the simulation cell in an almost straight line. 


\subsection{Determining sedimentation lengths}

To determine the sedimentation lengths of the squirmers, we need to be sure that the system is in a steady state. In the beginning of the simulations we initialize the squirmers with random positions and orientations. We then observe that the collection of squirmers "collapses" towards the bottom wall by monitoring the mean squirmer height $\langle z\rangle$. In continuing the simulations, we ensure that ultimately $\langle z\rangle$ does not show any deterministic trend, but is only subject to fluctuations. We then simulate for a period of at least $10^{4}$ MPCD time units (i.e. $10^{4} a_{0} \sqrt{m_{0} / k_{\mathrm{B}} T}$ ) and use this simulation data for our further analysis.

As explained in the results section, we determine the sedimentation or density profile $\rho(z)$ of the squirmers, from which we identify some layering at the bottom wall of the simulation box, which is followed by a transitional and then an exponential region. In the latter we determine the sedimentation length $\delta$ using an exponential fit. The difficulty is to specify a range of heights $\left[z_{\mathrm{b}}, z_{\mathrm{t}}\right]$, in which the exponential fit is performed. We have developed heuristic but robust criteria to identify this range. They ensure that neither layering at the bottom wall nor accumulation of squirmers at the top wall influences the fit values for $\delta$. As a first constraint we demand that $z_{\mathrm{t}}$ is at least a distance of $10 R$ away from the top wall. For $z_{\mathrm{b}}$ we require twice the height, which the squirmers would assume if they were all perfectly stacked in a hexagonal close packing. Within this first specification for the range $\left[z_{\mathrm{b}}, z_{\mathrm{t}}\right]$ we then determine the final $z_{\mathrm{t}}$ as the height, where the density $\rho$ is minimal. For $z_{\mathrm{b}}$ we take the smallest height $z$, where $\rho(z)$ falls below $8 \%$ of the hexagonal-close-packed density. The value of $8 \%$ is a purely empirical value.

We use the data in the range $\left[z_{\mathrm{b}}, z_{\mathrm{t}}\right]$ to obtain the sedimentation length $\delta$ from exponential fitting. In order to also estimate its error, we need to generate several estimates for $\delta$ from our data. Therefore, we split up the simulation time after reaching steady state into ten intervals. For each interval we perform exponential fits in four different ranges: (i) $\left[z_{\mathrm{b}}, z_{\mathrm{t}}\right]$, (ii) $\left[z_{\mathrm{b}}+0.1 \Delta z, z_{\mathrm{t}}\right]$, (iii) $\left[z_{\mathrm{b}}, z_{\mathrm{t}}-0.1 \Delta z\right]$, and (iv) $\left[z_{\mathrm{b}}+0.1 \Delta z, z_{\mathrm{t}}-0.1 \Delta z\right]$, where $\Delta z:=z_{\mathrm{t}}-z_{\mathrm{b}}$. We use the modified ranges as an additional measure to ensure that we are in the exponential regime. As an estimate for $\delta$, we then take the mean of all $40 \mathrm{fits}$, while the corresponding standard deviation specifies the error.

\section{Results}

After a transient, the squirmers under gravity settle into a steady state, which we analyze in the following. Fig. 1 shows a snapshot for a simulation, where the ratio of swimming to sedimentation velocity was $\alpha=1.0$. In the lower part layers of squirmers have formed. In particular, the lowest layers display clusters of hexagonal packing (see inset of Fig. 1), which gradually dissolves when moving upward. After a transition region, where layering is not recognizable anymore, a dilute region of squirmers follows, where we will identify the exponential density profile. In the ESI, $\dagger$ the two Videos V1 and V2 (for $\alpha=1.5$ ) illustrate very impressively how dynamic the whole sedimentation

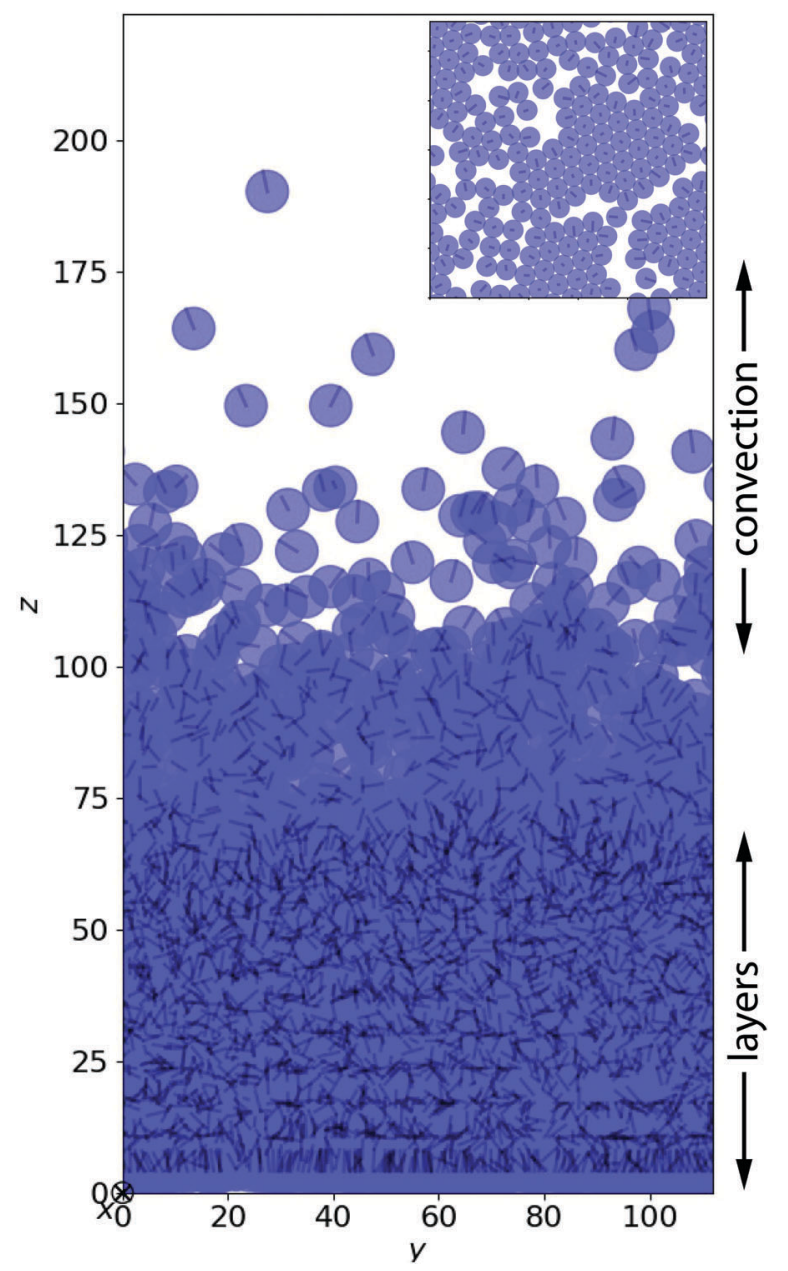

Fig. 1 Snapshot of 2560 neutral squirmers $(\beta=0)$ moving under gravity in a steady state at $\alpha=1$. The volume fraction is $\phi \approx 0.244$. Three regions can be distinguished: layering at the bottom, followed by a transitional regime, and finally a region with an exponential density profile and convective motion of squirmers. Inset: Hexagonal clustering in the lowest layer of squirmers.

profile is, especially in the exponential regime. This is in stark contrast to passive particles. In the following we will investigate some features of sedimenting squirmers in more detail.

\subsection{Sedimentation profile and vertical alignment}

The sedimentation profile in Fig. 2 quantifies the observations from Fig. 1. Close to the bottom, layering is clearly observable in the volume fraction or density $\rho(z)$ and indicated by peaks, the height of which gradually decreases within $c a .11$ layers. After a transitional regime, $\rho(z)$ decays exponentially and then is influenced by the upper bounding wall. Passive Brownian particles with buoyant mass $m$ show an exponential sedimentation profile with sedimentation length $\delta_{0}=k_{\mathrm{B}} T / m g$, as previously introduced. For very dilute suspensions of active particles, one can still derive $e^{2,18,20-22,67}$ and observe ${ }^{18,19}$ an exponential profile, however, with an increased sedimentation length $\delta>\delta_{0}$. Even if passive particles all sink to the bottom due to their weight $\left(\delta_{0} \ll R\right)$, active particles with sufficiently large swimming speed 
can rise from the bottom with a sedimentation length $\delta>R=4$. In our simulations, squirmers strongly interact hydrodynamically by the flow fields they generate. Nevertheless, we observe an exponential decay of the density $\rho(z)$ similar to lattice-Boltzmann simulations ${ }^{12,68}$ and experiments, ${ }^{18,19}$ which we find non-trivial. Thus from fits to the exponential part of the density profile, $\rho(z) \sim \mathrm{e}^{-z / \delta}$, we extract the sedimentation length $\delta$.

In Fig. 3(a) we present sedimentation profiles for a larger swimming speed, $\alpha=1.5$, and different swimmer types $\beta$ to explore the influence of pushers and pullers. At larger $\alpha$, all profiles show an exponential regime with a larger sedimentation length compared to $\alpha=1$. Fig. 4 shows a parametric study of $\delta$ in units of squirmer radius $R$ plotted versus $\beta$ and for three values of $\alpha$. Clearly, $\delta$ decreases with $\alpha$ and is only a fraction of $R$ for $\alpha=0.3$, when the activity is too small for particles to swim upwards. However, already the ratio $\alpha=1.5$ is sufficient to have sedimentation lengths $\delta \approx 10 R$. To address the robustness of

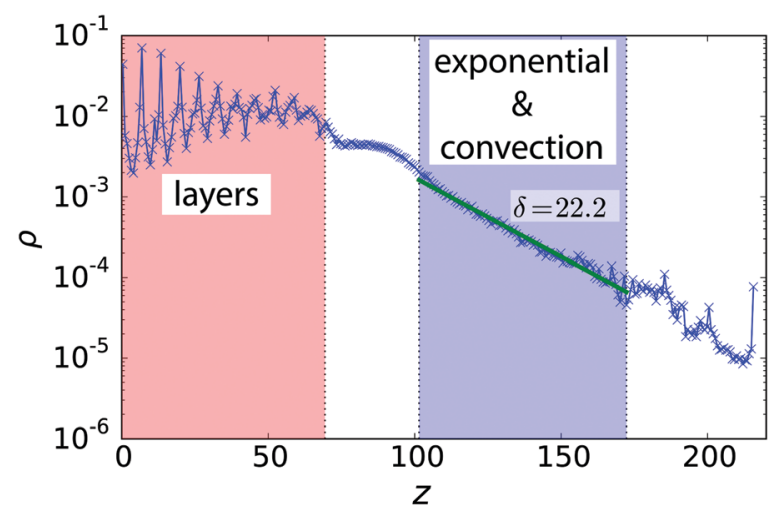

Fig. 2 Semi-logarithmic plot of the sedimentation profile $\rho(z)$ for the system in Fig. $1(\alpha=1.0$ and $\beta=0)$. Different regions are indicated. The green line is an exponential fit to extract the sedimentation length $\delta$. our results, for the case $\beta=0$ and $\alpha=1.5$, we reduced the volume density $\phi$ by decreasing the number of squirmers $N$ to a value where layer formation does not occur, while keeping the height $L_{z}$ of the simulation box fixed. This does not influence the sedimentation length $\delta$ significantly, as long as the squirmer density at the bottom of the box is similar to that of the top layers. For $\beta=0$ we also reduced both $N$ and $L_{z}$ by a factor of two, which keeps $\phi$ constant. We find that the sedimentation length is reduced by about $30 \%$ and $40 \%$ for $\alpha=1$ and $\alpha=1.5$, respectively. This is not surprising, since hydrodynamic interactions with the top wall, which were not relevant before, push squirmers downwards and also turn them away from the wall, ${ }^{58,69}$ which makes them swim downwards.

In Fig. 4 we realize that for weak pushers $(\beta=-1)$ the sedimentation length is the largest and decreases for stronger pushers and also pullers. It has been reported in the literature that the interaction between parallel squirmers grows with $|\beta|{ }^{48,70}$ We speculate that the collective interactions of many squirmers will therefore strongly randomize swimming directions and hinder squirmers with large $|\beta|$ from reaching larger heights, as reflected in the sedimentation length. The larger sedimentation length for weak pushers as compared to neutral squirmers is, however, unexpected. A possible explanation comes from the shape of flow fields for $\beta \neq 0$. Pushers, in their center-of-mass frame, have a stagnation point with vortical flow in front of them, while pullers have it at their back. ${ }^{23}$ Since squirmers are typically pointing up in the exponential regime (see below) and since their density $\rho(z)$ decreases with height $z$, pullers reorient more nearby squirmers compared to pushers, which decreases $\delta$. Interestingly, the trend of $\delta$ for varying $\beta$ is inverted for small $\alpha$ with the minimum being at $\beta \approx 1$. The reason for the inversion is not clear, but since $\delta<R$, we assume that interactions with the densely packed squirmer layers are relevant.

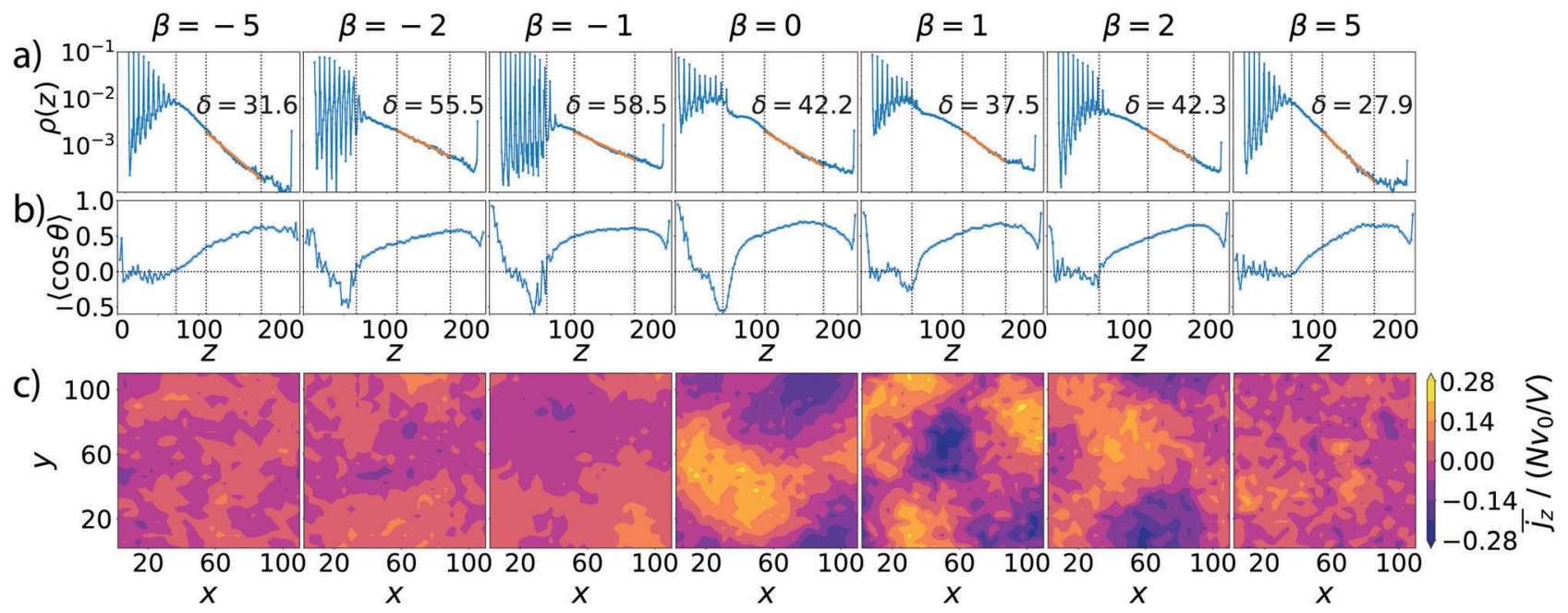

Fig. 3 Parameter study for varying $\beta$ at $\alpha=1$.5. (a) Semi-logarithmic plot of the squirmer density $\rho(z)$ as a function of height $z$. Vertical dashed lines in each plot indicate (from left to right) top of layering, followed by the start and end of the exponential regime, where the sedimentation length $\delta$ is extracted by an exponential fit (bold orange line). (b) Mean vertical orientation of squirmers as a function of height $z$. $\theta$ denotes the angle between the vertical and the squirmer orientation. (c) Vertical squirmer current density $\overline{j_{z}(\mathbf{x})}$, averaged over the exponential regime and time, color-coded in the $x y$ plane. 


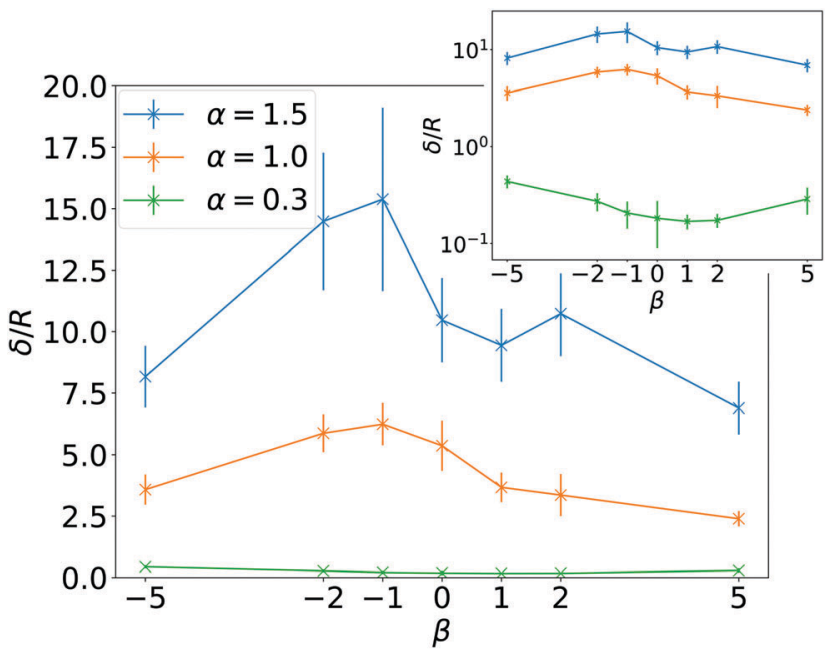

Fig. 4 Sedimentation length $\delta$ in units of $R$ as a function of squirmer parameter $\beta$ for different ratios of swimming to sedimentation velocities, $\alpha$. Inset: Semi-logarithmic plot.

In Fig. 3(a) we also observe how the layer structure in the lower part of the system is influenced by $\beta$. For $\beta=0$ and $\beta=1$ the minima between successive layers are less pronounced, which implies less order. In contrast, especially for $\beta=-1$ and $\beta=-2$ layering is more pronounced. We ascribe this difference to the hydrodynamic interactions between neighboring squirmers, which depend on $\beta$.

Finally, in Fig. 5 we show the mean orientation of squirmers as a function of height $z$ for the system illustrated in Fig. 1. The neutral squirmers in the bottom layer at $z=0$ have an upright orientation due to hydrodynamic interactions with the bounding wall. ${ }^{31,41,42,48}$ The mean orientation then decreases to zero (see also Fig. 1) and drops to a negative value at the rim of the layering. This is simply because squirmers from above swim into the dense squirmer region and need some time to reorient and swim away. In the transitional region the orientation changes again rapidly to nearly upright and shows only small variations in the exponential regime.

The occurrence of polar order in the sedimentation profile of dilute suspensions has been predicted by theory and already

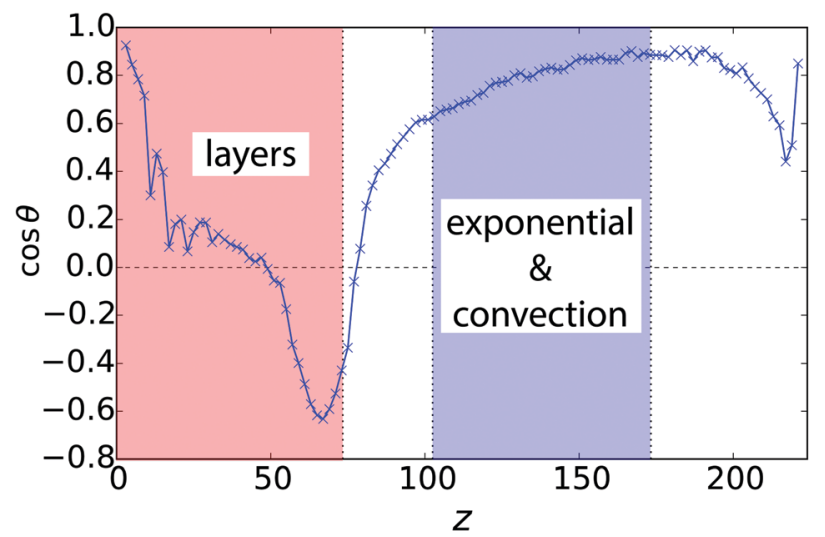

Fig. 5 Mean vertical orientation of squirmers, $\langle\cos \theta\rangle$, as a function of height $z$ for the system in Fig. $1(\alpha=1.0$ and $\beta=0$ ). occurs without any interactions (i.e. for dilute suspensions of active Brownian particles) just for kinetic reasons. ${ }^{2,21}$ Hydrodynamic interactions between squirmers obviously do not destroy the polar order. In our parametric study of Fig. 3(c), this is also confirmed for other squirmer types $\beta$. Differences occur in the layering and in the transitional region. For pullers the upright orientation in the bottom layer decreases for larger $\beta$, as expected by hydrodynamic interactions with the bottom wall in lubrication theory. ${ }^{41,42,48}$ In the adjacent layers hardly any polar order is visible in contrast to neutral squirmers. Weak pushers $(\beta=-1$ and -2$)$ show a similar but weaker trend compared to neutral squirmers. For strong pushers $(\beta=-5)$, again, there is hardly any polar order in the layering.

\subsection{Convection}

As Videos V1 and V2 in the ESI $\dagger$ demonstrate, the squirmers in the exponential density region are very mobile. In fact, while their mean vertical velocity is zero, the steady-state distribution of vertical velocities for $\beta=0$ and $\alpha=1.5$ can be well fitted by a Gaussian with a standard deviation comparable to $v_{0}$ (see Fig. 6). For large $|\beta|$ small deviations from the Gaussian form occur. Thus, also vertical squirmer speeds larger than $\frac{1}{3} v_{0}$ arise (the maximal vertical velocity a single bulk squirmer can have at $\alpha=1.5$ ). This means that squirmers are advected by flow fields set up by their neighbors. Indeed, for neutral squirmers and weak pullers/pushers, we see evidence for convection flow extending over the whole simulation cell.

To quantify convection, we take the vertical squirmer current density and average it along the vertical in the exponential regime:

$$
j_{z}(\mathbf{x})=\langle\rho\rangle_{\|}(\mathbf{x})\left\langle v_{z}\right\rangle_{\|}(\mathbf{x})
$$

where $\mathbf{x}=(x, y)$ is a position in the horizontal plane, $\langle\cdots\rangle_{\|}$means average along the vertical, and $\rho$ is the squirmer density. In the following we always indicate a time average over some quantity $q$ in the steady state by $\bar{q}$. We plot $\overline{j_{z}(\mathbf{x})}$ in Fig. 7 for neutral squirmers and $\alpha=1.5$ in the $x y$ plane of the simulation box.

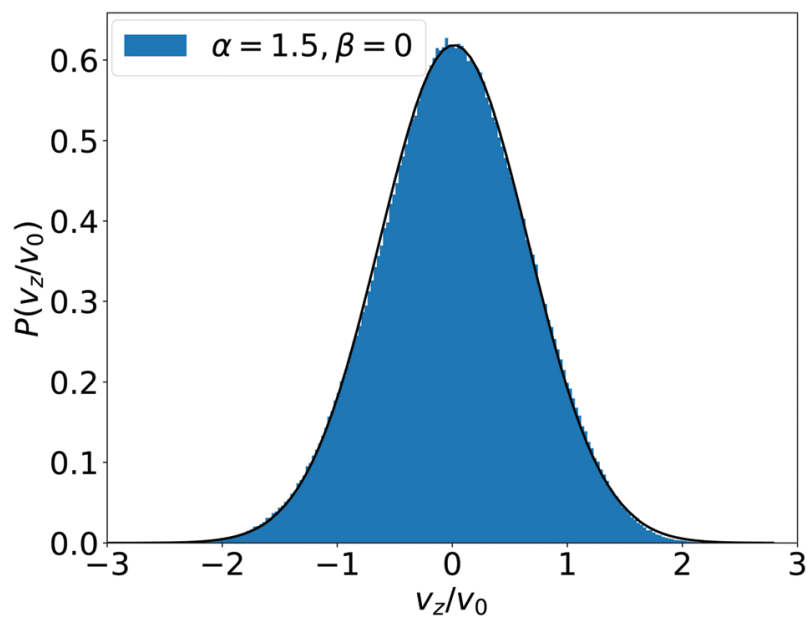

Fig. 6 Distribution of vertical squirmer velocity (blue) and Gaussian fit (black) for $\alpha=1.5$ and $\beta=0$ in the exponential regime. 


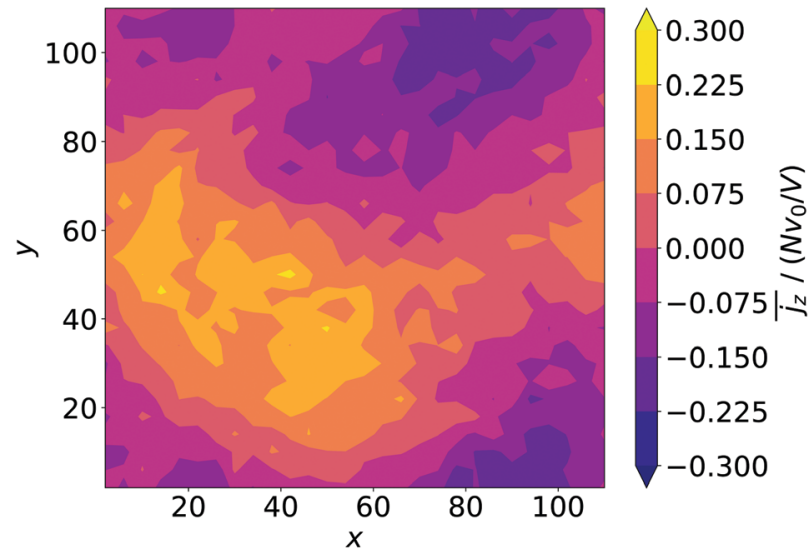

Fig. 7 Squirmer current density, $\overline{j_{z}(\mathbf{x})}$, averaged over the exponential regime and time, color-coded in the $x y$ plane for $\alpha=1.5$ and $\beta=0$.

While in the lower left region squirmers move upward, the vertical current goes downward in the upper right indicating a convection cell, which extends over the whole horizontal plane. In Fig. 3(c) we present $\overline{j_{z}(\mathbf{x})}$ for different squirmer types at the same $\alpha$. For weak pullers $(\beta=1$ and 2$)$ the extent of the convection cell decreases and at $\beta=5$ large-scale convection is no longer observable. For weak pushers $(\beta=-1$ and -2$)$ the current density becomes weaker but still large-scale convection is visible, which then vanishes for $\beta=-5$. For the case $\beta=0$, $\alpha=1.5$ we checked that large-scale convection is stable against a reduction in the squirmer density $\phi$ at constant $L_{z}$ and for reduced $L_{z}$ while keeping $\phi$ constant. In both settings a single convection cell extends across the simulation box.

To further characterize the squirmer density current $j_{z}(\mathbf{x})$, we calculate its zeroth and first moment. The zeroth moment is the volume average of the vertical current density:

$$
\left\langle j_{z}\right\rangle=\frac{1}{A} \int_{A} j_{z}(\mathbf{x}) \mathrm{d}^{2} x=\langle\rho\rangle\left\langle v_{z}\right\rangle,
$$

where $A$ is the area of the $x y$ plane of the simulation cell and $\langle\cdots\rangle$ means average over the whole volume of the exponential region. The vertical current density $\left\langle j_{z}\right\rangle$ strongly fluctuates in time (see Fig. 8) but in the steady state its temporal mean, $\overline{\left\langle j_{z}\right\rangle}$, has to be zero because of particle conservation. Thus, we use $\left\langle\left|j_{z}\right|\right\rangle$ to quantify how mobile the squirmers are in the exponential region (see Fig. 8). In Fig. 9 we show the time average $\overline{\left\langle\left|j_{z}\right|\right\rangle}$ versus $\beta$ for different rescaled swimming velocities $\alpha$. As expected, at $\alpha>1$ the squirmers are more mobile than for $\alpha \leq 1$ since they are able to move against gravity. Furthermore, for $\alpha=1.5$ the mean vertical current density $\overline{\left\langle\left|j_{z}\right|\right\rangle}$ decreases for large $|\beta|$, revealing again the importance of the advective flow set up by the different squirmer types.

We call the first moment of $j_{z}(\mathbf{x})$ current dipole,

$$
\mathbf{j}_{\mathrm{D}}=\frac{1}{A} \int_{A} \mathbf{x} j_{z}(\mathbf{x}) \mathrm{d}^{2} x,
$$

where we choose the center of the $x y$ plane as the origin of $\mathbf{x}$. The current dipole serves to quantify the strength and horizontal

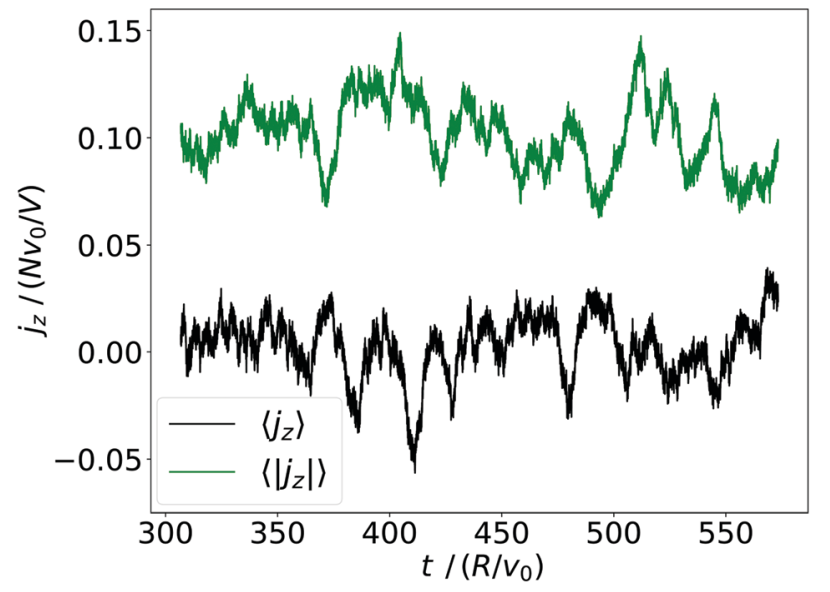

Fig. 8 Volume average of the squirmer current density, $\left\langle j_{z}\right\rangle$, and its magnitude, $\left\langle\left|j_{z}\right|\right\rangle$, plotted versus time for $\alpha=1.5$ and $\beta=0$.

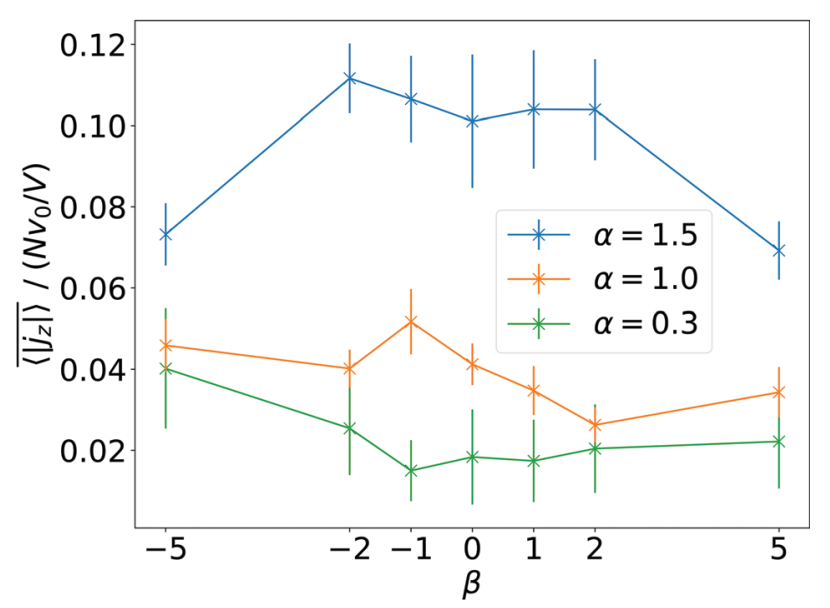

Fig. 9 Time and volume average of the magnitude of the current density, $\overline{\left\langle\left|j_{z}\right|\right\rangle}$, as a function of squirmer parameter $\beta$ for different rescaled swimming speeds $\alpha$.

extension of the convection cell by its magnitude $j_{\mathrm{D}}:=\left|\mathbf{j}_{\mathrm{D}}\right|$. The cell's orientation relative to the $x$-axis is given by the angle $\varphi_{\mathrm{D}}$ with $\cos \varphi_{\mathrm{D}}=\mathbf{j}_{\mathrm{D}} \cdot \mathbf{e}_{x} / j_{\mathrm{D}}$. Fig. 10 shows how $j_{\mathrm{D}}$ strongly fluctuates in time, reflecting again the high mobility of the squirmers. The orientation angle $\varphi_{\mathrm{D}}$ also fluctuates and in the example of Fig. 10 assumes two mean orientations around $1.5 \pi$ and $\pi$. Overall, we can record that the spatial arrangement of convection is subject to strong fluctuations and is strongly variable in time.

Finally, in Fig. 11 we plot the time average $\overline{j_{\mathrm{D}}}$ versus squirmer type $\beta$ for different $\alpha$. Convection is largest for large $\alpha$ and neutral squirmers. The current dipole we define in eqn (5) can be interpreted in analogy with a charge dipole in electrostatics. Its magnitude changes when either the current density (the separated "charges") changes in magnitude or when the distance between regions of positive vs. negative vertical speed is altered (the distance of "charge separation"). In accordance with Fig. 3(c) we find that $\overline{j_{\mathrm{D}}}$ for $\alpha=1.5$ decreases when the magnitude of the current density $j_{z}(\mathbf{x})$ decreases (weak pusher, $\beta<0$ ) or the extension of the convection cell becomes smaller (weak puller, $\beta>0$ ). 


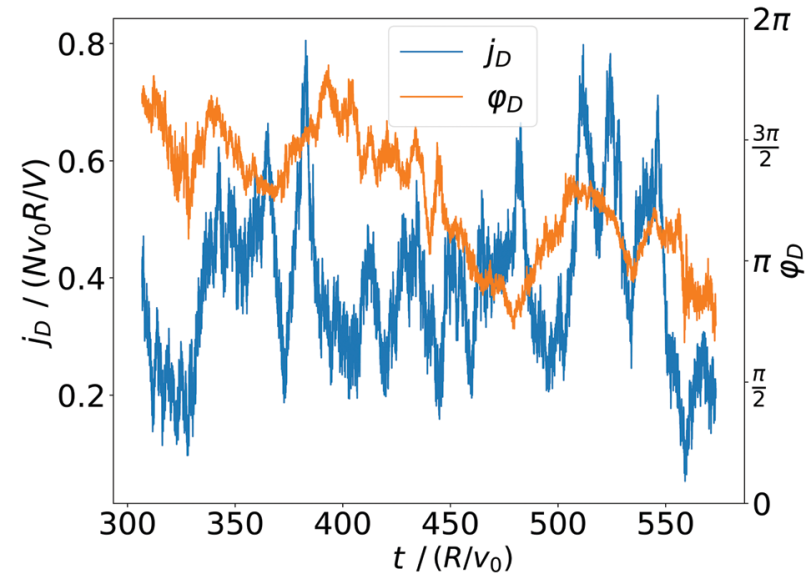

Fig. 10 Magnitude $j_{D}$ and orientation angle $\varphi_{D}$ of the current dipole plotted versus time for $\alpha=1.5$ and $\beta=0$.

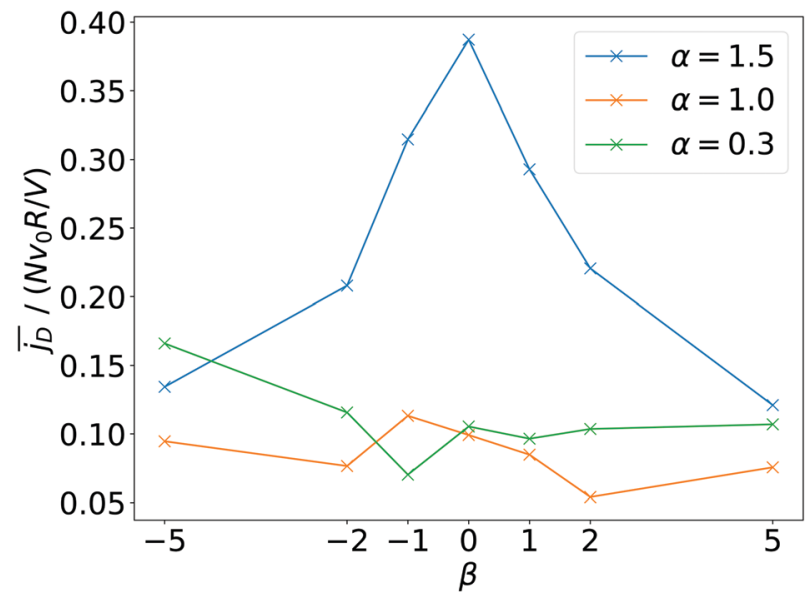

Fig. 11 The time average of the magnitude of the current dipole, $\overline{j_{\mathrm{D}}}$, as a function of squirmer parameter $\beta$ for different rescaled swimming speeds $\alpha$.

Strong pushers/pullers with $|\beta|=5$ only show weak convection. For small rescaled swimming speed $\alpha \leq 1$ convection is generally small.

\section{Conclusions}

In this work we addressed the collective sedimentation of squirmers in a gravitational field concentrating on the relevant case, where the corresponding passive particles would completely sediment. We showed that the sedimentation profile can be divided into three distinct regions; closely packed layers near the bottom, a transition region, and a rather dilute active suspension at the top. Here, the density depends exponentially on height, as for passive colloids, and the profile is very dynamic. The exponential dependence is non-trivial given the strong hydrodynamic interactions between the squirmers due to their flow fields. We also identified a strongly height-dependent mean orientation of the swimmers. While the mean orientation is strongly varying across the closely packed layers, in particular for small $|\beta|$, it varies far less in the exponential region. From the latter we extracted sedimentation lengths and showed that these not only grow with the ratio of active to sedimentation velocity, but also depend on the squirmer type. We argue that neutral squirmers or weak pushers and pullers are more persistent when swimming upwards and thereby show larger sedimentation lengths.

Furthermore, sedimenting squirmers create strong convective currents due to their hydrodynamic interactions. The spatial extension and the strength of convection are again determined by the rescaled swimming speed and by the squirmer type. Neutral squirmers as well as weak pushers and pullers show the strongest convectional flow. In particular, for swimming speeds larger than the sedimentation velocity pronounced convection cells occur, which extend over the whole simulation box. Finally, as another signature of the highly dynamic sedimentation profile in the exponential region, we identified strong temporal fluctuations of the convective currents.

What we could not resolve in our current simulations due to limited computational resources is the question of what determines the lateral extent of the convection cells. Is there an intrinsic length scale, which sets it? For this we would need to increase the simulation cell in the lateral directions. In future work, we plan to include bottom heaviness of the squirmers in our simulations and study in detail the inversion of the sedimentation profile, which was discussed in ref. 22 for very dilute swimmer suspensions. Preliminary results in denser systems show that instabilities occur due to hydrodynamic interactions. ${ }^{71}$ In a harmonic trapping potential a similar instability leads to the formation of fluid pumps by breaking the rotational symmetry of the trap. ${ }^{12,14}$ In the present case we expect convectional patterns to occur. Thereby, we will connect to the phenomenon of bioconvection. ${ }^{36-39}$

\section{Conflicts of interest}

There are no conflicts to declare.

\section{Acknowledgements}

We would like to thank C. Cottin-Bizonne and A. Zöttl for stimulating discussions. This project was funded by Deutsche Forschungsgemeinschaft through the research training group GRK 1558 and priority program SPP 1726 (grant number STA352/11). Simulations were conducted at the "Norddeutscher Verbund für Hoch- und Höchstleistungsrechnen" (HLRN), project number bep00050.

\section{References}

1 E. Lauga and T. R. Powers, Rep. Prog. Phys., 2009, 72, 096601. 2 H. Stark, Eur. Phys. J.: Spec. Top., 2016, 225, 2369-2387.

3 A. Sokolov and I. S. Aranson, Phys. Rev. Lett., 2009, 103, 148101. 4 S. Rafa, L. Jibuti and P. Peyla, Phys. Rev. Lett., 2010, 104, 600. 5 H. M. López, J. Gachelin, C. Douarche, H. Auradou and E. Clément, Phys. Rev. Lett., 2015, 115, 121.

6 E. Clément, A. Lindner, C. Douarche and H. Auradou, Eur. Phys. J.: Spec. Top., 2016, 225, 2389-2406. 
7 A. Zöttl and H. Stark, Phys. Rev. Lett., 2012, 108, 218104.

8 S. Uppaluri, N. Heddergott, E. Stellamanns, S. Herminghaus, A. Zöttl, H. Stark, M. Engstler and T. Pfohl, Biophys. J., 2012, 103, 1162-1169.

9 A. Zöttl and H. Stark, Eur. Phys. J. E: Soft Matter Biol. Phys., 2013, 36, 3.

10 R. Rusconi, J. S. Guasto and R. Stocker, Nat. Phys., 2014, 10, 212-217.

11 M. Tarama, A. M. Menzel and H. Löwen, Phys. Rev. E: Stat., Nonlinear, Soft Matter Phys., 2014, 90, 032907.

12 R. W. Nash, R. Adhikari, J. Tailleur and M. E. Cates, Phys. Rev. Lett., 2010, 104, 258101.

13 A. Pototsky and H. Stark, Europhys. Lett., 2012, 98, 50004.

14 M. Hennes, K. Wolff and H. Stark, Phys. Rev. Lett., 2014, 112, 238104.

15 A. M. Menzel, A. Saha, C. Hoell and H. Löwen, J. Chem. Phys., 2016, 144, 024115.

16 J. A. Cohen and R. Golestanian, Phys. Rev. Lett., 2014, 112, 365.

17 C. Lozano, B. ten Hagen, H. Löwen and C. Bechinger, Nat. Commun., 2016, 7, 12828.

18 J. Palacci, C. Cottin-Bizonne, C. Ybert and L. Bocquet, Phys. Rev. Lett., 2010, 105, 088304.

19 F. Ginot, I. Theurkauff, D. Levis, C. Ybert, L. Bocquet, L. Berthier and C. Cottin-Bizonne, Phys. Rev. X, 2015, 5, 011004.

20 J. Tailleur and M. E. Cates, Europhys. Lett., 2009, 86, 60002. 21 M. Enculescu and H. Stark, Phys. Rev. Lett., 2011, 107, 058301.

22 K. Wolff, A. M. Hahn and H. Stark, Eur. Phys. J. E: Soft Matter Biol. Phys., 2013, 36, 9858.

23 A. A. Evans, T. Ishikawa, T. Yamaguchi and E. Lauga, Phys. Fluids, 2011, 23, 111702.

24 M. C. Marchetti, J. F. Joanny, S. Ramaswamy, T. B. Liverpool, J. Prost, M. Rao and R. A. Simha, Rev. Mod. Phys., 2013, 85, 1143-1189.

25 D. Saintillan and M. J. Shelley, C. R. Phys., 2013, 14, 497-517.

26 F. Alarcón and I. Pagonabarraga, J. Mol. Liq., 2013, 185, 56-61.

27 W. Yan and J. F. Brady, Soft Matter, 2015, 11, 6235-6244.

28 A. Zöttl and H. Stark, J. Phys.: Condens. Matter, 2016, 28, 253001.

29 C. Krüger, C. Bahr, S. Herminghaus and C. C. Maass, Eur. Phys. J. E: Soft Matter Biol. Phys., 2016, 39, 103.

30 M. E. Cates and J. Tailleur, Annu. Rev. Condens. Matter Phys., 2015, 6, 219-244.

31 A. Zöttl and H. Stark, Phys. Rev. Lett., 2014, 112, 118101.

32 R. Matas-Navarro, R. Golestanian, T. B. Liverpool and S. M. Fielding, Phys. Rev. E: Stat., Nonlinear, Soft Matter Phys., 2014, 90, 032304.

33 R. M. Navarro and S. M. Fielding, Soft Matter, 2015, 11, 7525-7546.

34 J. Blaschke, M. Maurer, K. Menon, A. Zöttl and H. Stark, Soft Matter, 2016, 12, 9821-9831.

35 N. Oyama, J. J. Molina and R. Yamamoto, Phys. Rev. E: Stat., Nonlinear, Soft Matter Phys., 2016, 93, 043114.

36 H. Wager, Philos. Trans. R. Soc., B, 1911, 201, 333.

37 T. J. Pedley and J. O. Kessler, Annu. Rev. Fluid Mech., 1992, 24, 313-358.
38 C. R. Williams and M. A. Bees, J. Exp. Biol., 2011, 214, 2398-2408. 39 C. R. Williams and M. A. Bees, J. Fluid Mech., 2011, 678, 41-86. 40 I. Llopis and I. Pagonabarraga, J. Non-Newtonian Fluid Mech., 2010, 165, 946-952.

41 K. Schaar, A. Zöttl and H. Stark, Phys. Rev. Lett., 2015, 115, 038101. 42 J. S. Lintuvuori, A. T. Brown, K. Stratford and D. Marenduzzo, Soft Matter, 2016, 12, 7959-7968.

43 F. Rühle, J. Blaschke, J.-T. Kuhr and H. Stark, New J. Phys., submitted.

44 A. Malevanets and R. Kapral, J. Chem. Phys., 1999, 110, 8605.

45 G. Gompper, T. Ihle, D. M. Kroll and R. G. Winkler, Advances in polymer science 221, Springer, Berlin, 2009, pp. 1-87.

46 M. J. Lighthill, Comm. Pure Appl. Math., 1952, 5, 109-118.

47 J. R. Blake, J. Fluid Mech., 1971, 46, 199.

48 T. Ishikawa, M. P. Simmonds and T. J. Pedley, J. Fluid Mech., 2006, 568, 119-160.

49 M. T. Downton and H. Stark, J. Phys.: Condens. Matter, 2009, 21, 204101.

50 R. Golestanian, T. B. Liverpool and A. Ajdari, Phys. Rev. Lett., 2005, 94, 53 .

51 S. Ebbens, D. A. Gregory, G. Dunderdale, J. R. Howse, Y. Ibrahim, T. B. Liverpool and R. Golestanian, Europhys. Lett., 2014, 106, 58003.

52 S. Thutupalli, R. Seemann and S. Herminghaus, New J. Phys., 2011, 13, 073021.

53 M. Schmitt and H. Stark, Europhys. Lett., 2013, 101, 44008.

54 M. Schmitt and H. Stark, Eur. Phys. J. E: Soft Matter Biol. Phys., 2016, 39, 062901.

55 M. Schmitt and H. Stark, Phys. Fluids, 2016, 28, 012106.

56 C. C. Maass, C. Krüger, S. Herminghaus and C. Bahr, Annu. Rev. Condens. Matter Phys., 2016, 7, 171-193.

57 C. Jin, C. Krüger and C. C. Maass, Proc. Natl. Acad. Sci. U. S. A., 2017, 114, 5089-5094.

58 S. E. Spagnolie and E. Lauga, J. Fluid Mech., 2012, 700, 105-147.

59 J. T. Padding and A. A. Louis, Phys. Rev. E: Stat., Nonlinear, Soft Matter Phys., 2006, 74, 031402.

60 H. Noguchi, N. Kikuchi and G. Gompper, Europhys. Lett., 2007, 78, 10005.

61 R. Kapral, Advances in Chemical Physics 140, John Wiley \& Sons, Hoboken, 2008, pp. 89-146.

62 J. T. Padding, A. Wysocki, H. Löwen and A. A. Louis, J. Phys.: Condens. Matter, 2005, 17, S3393.

63 J. T. Padding and W. J. Briels, J. Chem. Phys., 2010, 132, 054511. 64 P. Kanehl and H. Stark, J. Chem. Phys., 2015, 142, 214901. 65 P. Kanehl and H. Stark, Phys. Rev. Lett., 2017, 119, 018002. 66 H. Noguchi and G. Gompper, Phys. Rev. E: Stat., Nonlinear, Soft Matter Phys., 2008, 78, 016706.

67 J. Tailleur and M. E. Cates, Phys. Rev. Lett., 2008, 100, 218103. 68 M. E. Cates, K. Stratford, R. Adhikari, P. Stansell, J.-C. Desplat, I. Pagonabarraga and A. J. Wagner, J. Phys.: Condens. Matter, 2004, 16, S3903.

69 A. P. Berke, L. Turner, H. C. Berg and E. Lauga, Phys. Rev. Lett., 2008, 101, 23.

70 I. O. Götze and G. Gompper, Phys. Rev. E: Stat., Nonlinear, Soft Matter Phys., 2010, 82, 041921.

71 M. Hennes, K. Wolff and H. Stark, unpublished results. 